\title{
Pollution level and source of cyanide in Jiaozhou Bay, eastern China
}

\section{Dongfang Yang ${ }^{1,2}$, , Xinhuai $\mathrm{He}^{1}$, Jie Gao ${ }^{1}$, Aijie Xin ${ }^{1}$ and Feijuan Wang ${ }^{1}$}

${ }^{1}$ College of medicine, Shaanxi institute of international trade \& commerce, Xian 712046, China;

${ }^{2}$ North China Sea Environmental Monitoring Center, SOA, Qingdao 266033, China

adfyang_dfyang@126.com

Keywords: Cyanide, Distribution, Source, Pollution level, Jiaozhou Bay.

\begin{abstract}
In order to provided background material of cyanide pollution history of Jiaozhou Bay, this paper analyzed the pollution and source of cyanide inJiaozhou Bay in 1982. Results showed that Jiaozhou Bay had not been polluted by cyanide in 1982. The contents of cyanide in June and October in surface waters ranged from $0.10-0.28 \mu \mathrm{g} . \mathrm{L}^{-1}$ and $0.02-0.31 \mu \mathrm{g} . \mathrm{L}^{-1}$, which could be considered as the background pollution level of cyanide in Jiaozhou Bay. Point source was the major source of cyanide in Jiaozhou Bay in dry season, while both point and non-point sources were the major sources in wet season.
\end{abstract}

\section{Introduction}

Cyanide (often refers to sodium cyanide and potassium cyanide) were widely used in gold production and electroplating industries [1-2]. Cyanide could be entered into human body by the way of respiratory tract, digestive tract and skin touch, combined with ferric iron of cytochrome oxidase, and resulted in the cutting of the oxygen transfer and the death of organism [3]. The wastes of the industries above were rich in cyanide, and had caused various environmental problems [4].

Jiaozhou Bay $\left(35^{\circ} 55^{\prime}-36^{\circ} 18^{\prime} \mathrm{N}, 120^{\circ} 04^{\prime}-120^{\circ} 23^{\prime} \mathrm{E}\right)$ is located in the south of Shandong Province, eastern China (Fig. 1). Being a semi-closed bay [5], the water exchange capacity of Jiaozhou Bay was relative low, and therefore understanding the pollution level and changing trend of cyanide is essential to environment protection.

This paper analyzed the distribution and source of cyanide in Jiaozhou Bay surface waters in 1982, and provided background material of cyanide pollution history of Jiaozhou Bay, which would be helpful to environmental assessment and remediation in this bay.

\section{Material and method}

The data was provided by North China Sea Environmental Monitoring Center. The survey was conducted in June and October 1982. Cyanide in surface waters was collected and measured followed by National Specification for Marine Monitoring [3]. For the investigation in June, there were four monitoring sites namely $\mathrm{H} 37, \mathrm{H} 39, \mathrm{H} 40$ and $\mathrm{H} 41$; while for October, there were five monitoring sites namely $083,084,121,122$ and 123. 


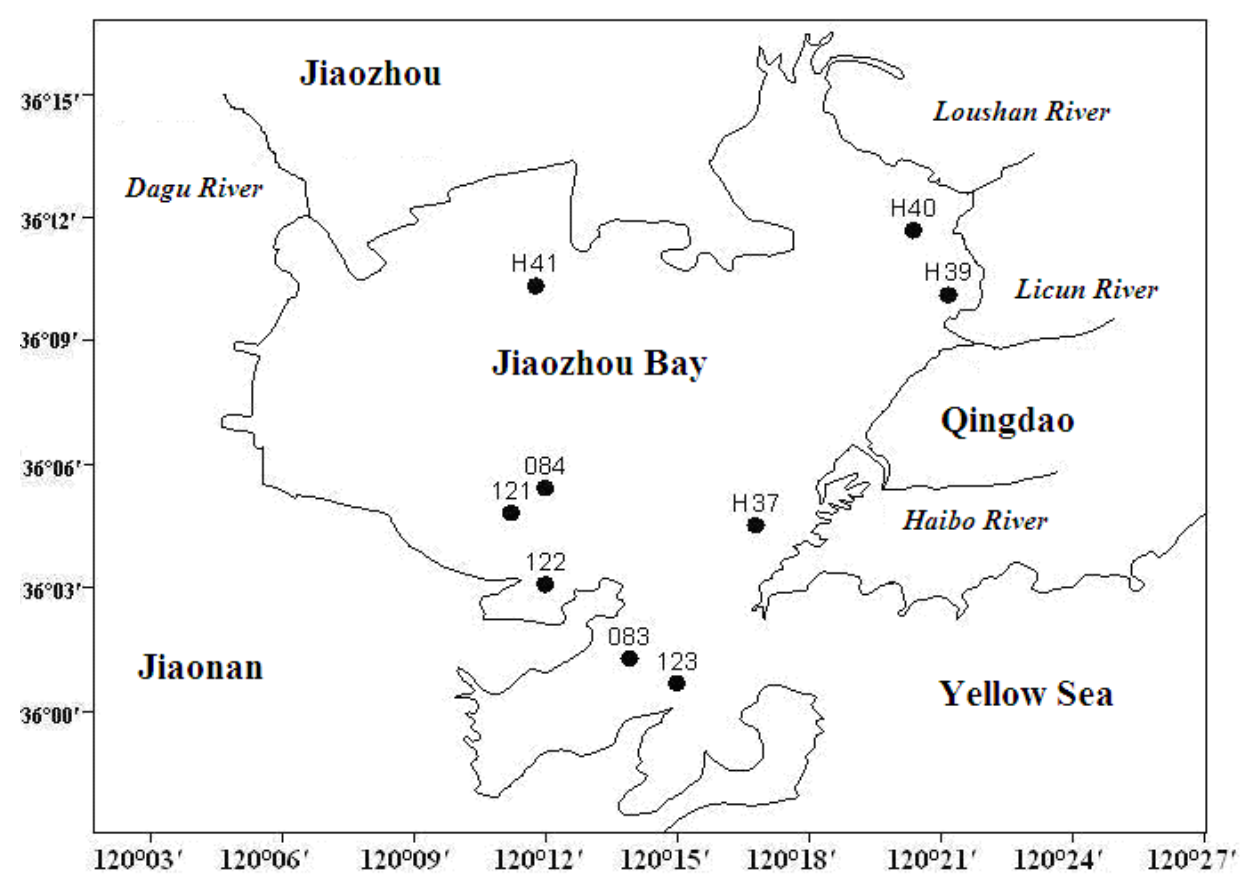

Fig.1 Monitoring sites in Jiaozhou Bay

\section{Results and discussion}

Pollution levels of cyanide. The criterion of cyanide in sea waters in Grade I in National Sea Water Quality Standard (GB 3097-1997) was $5.00 \mu \mathrm{g} \cdot \mathrm{L}^{-1}$. The contents of cyanide in June and October in surface waters ranged from $0.10-0.28 \mu \mathrm{g} . \mathrm{L}^{-1}$ and $0.02-0.31 \mu \mathrm{g} . \mathrm{L}^{-1}$, which were greatly lower than Grade I in GB 3097-1997. It could be conclude that Jiaozhou Bay had not been polluted by cyanide in 1982. Meanwhile, these content ranges of cyanide could be considered as the background pollution level of cyanide in Jiaozhou Bay.

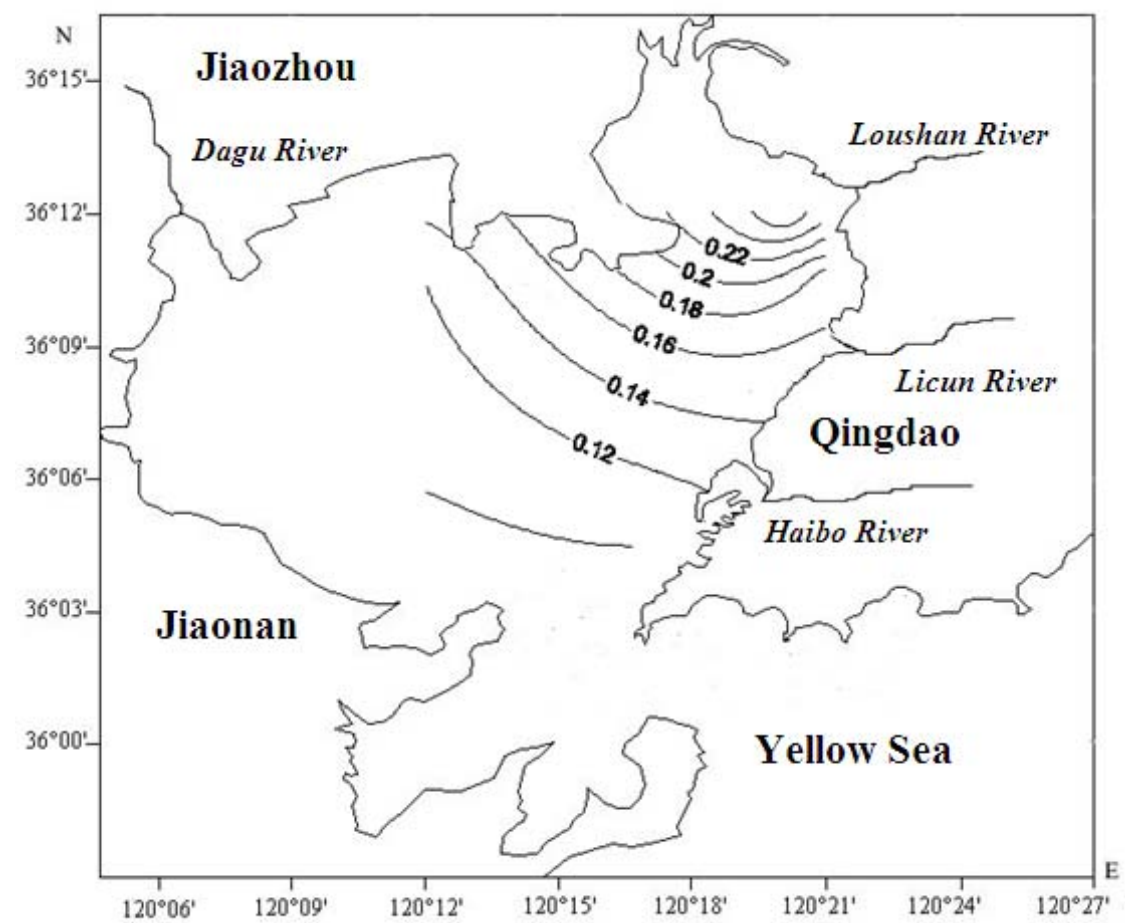

Fig. 2 Distribution of cyanide in surface in Jiaozhou Bay in June / $\mu \mathrm{g} . \mathrm{L}^{-1}$ 
Distributions of cyanide. In June, high value of cyanide contents occurred in Site H40 (0.28 $\left.\mu \mathrm{g} . \mathrm{L}^{-1}\right)$, which was closed to the river mouth of Loushan River, and the contents of cyanide were decreasing with the flow direction of these rivers (Fig. 2). In October, high value occurred in Site $121\left(0.31 \mu \mathrm{g} . \mathrm{L}^{-1}\right)$, which was located in southwest of the bay, and the contents of cyanide were decreasing were decreasing from the high value center to the bay mouth (Fig. 3). Obviously, the distribution trends of cyanide in June and October, on behalf of wet and dry season, were very different.

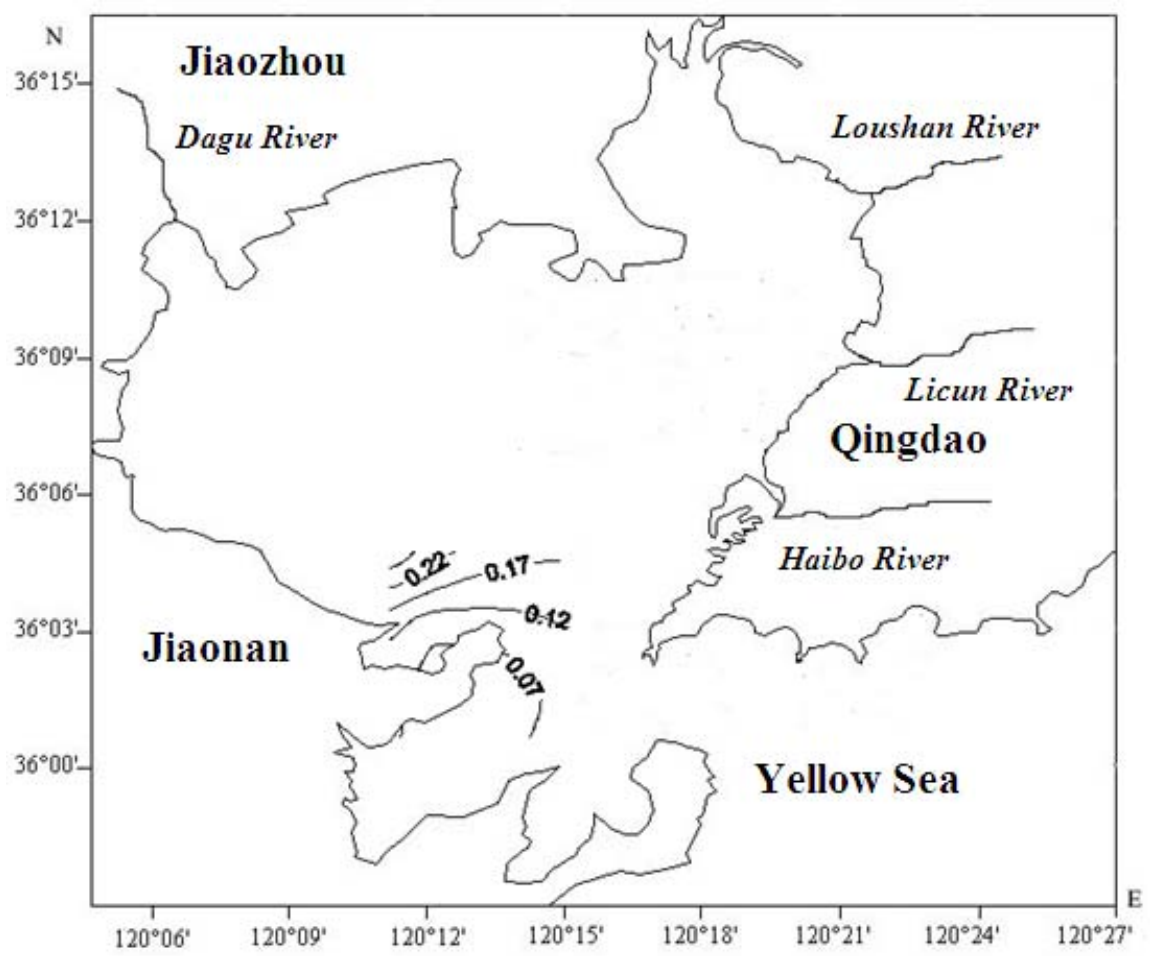

Fig. 3 Distribution of cyanide in surface in Jiaozhou Bay in June / $\mu \mathrm{g} . \mathrm{L}^{-1}$

Sources of cyanide. June and October could be categorized into wet and dry season in study area based on the amount of precipitation. In wet season relative more cyanide was washed from land surface, and was finally delivered to the bay via stream flows. Therefore, the cyanide contents in the bay were decreasing along with the flow direction of there rivers. While in dry season, the inputs of cyanide from the stream flows was limited due to the lack of precipitation, so there was no decreasing trend as in wet season. However, cyanide contents were decreasing from the southwest of the bay to the bay mouth, indicate that some small rivers located in Jiaonan Region in the southwest were the major input channels of cyanide. From the above, it could be concluded that point source was the major source of cyanide in Jiaozhou Bay in dry season, while both point and non-point sources were the major sources in wet season.

\section{Conclusion}

Jiaozhou Bay had not been polluted by cyanide in 1982, whose contents of cyanide in June and October in surface waters ranged from $0.10-0.28 \mu \mathrm{g} . \mathrm{L}^{-1}$ and $0.02-0.31 \mu \mathrm{g} . \mathrm{L}^{-1}$, which could be considered as the background pollution level of cyanide in Jiaozhou Bay. Point source was the major source of cyanide in Jiaozhou Bay in dry season, while both point and non-point sources were the major sources in wet season.

\section{Acknowledgement}

This paper was sponsored by Education Ministry's New Century Excellent Talents Supporting 
Plan (NCET-12-0659), Project of Outstanding Technological Educators of Governor of Guizhou ([2012]71), Project of Low Carbon Technology Plan of Guiyang (2012205]), Project of Science and Technology Foundation of Guiyang (LKM[2012]05), Special Research Projects of High Level

Talents of Guizhou Province (TZJF-2011-44), and Research Projects of Guizhou Nationalities University ([2014]02).

\section{References}

[1] Congbo Zhong, Chenggong Wang and Bingchen Chen: Metal Mine, Vol. 30 (2002) , p. 44-47. (in Chinese)

[2] Shehong Li, Baoshen Zheng, Jiaming Zhu and Binbin Wang: Environ. Sci., Vol. 22 (2001), p. 126-128. (in Chinese)

[3] Tao Zhang, Hao Qiu, Zou Zeli, Hanyang Dong, Zhihao Zhao, Xiang Wei, Weihua Zhang, Xinde Cai and Rongliang Qiu: Acta Sci. Circun., Vol. 29(2009), p. 1465-1469. (in Chinese)

[4] YipingJiang, Xiaoping Ao and Liangen Wang: Industrial Water \& Wastewater, Vol. 36(2006), p. 43-45. (in Chinese)

[5] Dongfang Yang, Yu Chen, Zhenhui Gao, Jing Zhang and Fan Wang: Chin. J. OceanoL. LimnoL., Vol. 23(2005), p. 72-90. 